Boll. Accademia Gioenia di

Scienze Naturali - Catania
BOLLAG Vol. 52, N. 382 (2019)

Difesa ecosostenibile delle colture agricole pp. DECA17 - DECA21

ISSN 0393-7143

Anno di fondazione 1824

\title{
Impiego di agenti biologici e mezzi biotecnici nella lotta contro gli insetti dannosi ${ }^{\dagger}$
}

\author{
Placido Reina [1]* \\ CBC Europe srl Biogard Division, Grassobio (Bergamo)
}

\begin{abstract}
Riassunto
Nel corso degli ultimi decenni, la difesa dai parassiti animali si è progressivamente orientata verso tecniche di difesa integrata, limitando l'uso del controllo chimico a vantaggio di tecniche di lotta alternative preferendo, laddove possibile, l'impiego di agenti e mezzi biologici. Alla luce delle acquisizioni scientifiche in campo nutrizionistico e soprattutto a seguito di una maggiore consapevolezza sociale, il mercato si sta sempre più orientando verso produzioni ortofrutticole con ridotta presenza di residui chimici. Ciò ha pertanto promosso la ricerca, lo sviluppo e la commercializzazione di prodotti fitosanitari efficaci ma con un profilo eco-tossicologico più favorevole. Largo impiego trovano formulati insetticidi basati su estratti vegetali e formulati microbiologici. Anche i feromoni sessuali si impiegano ampiamente sia in pieno campo che in coltura protetta (confusione e disorientamento sessuale), così come le tecniche di "attract \& kill" che sfruttano l'azione attrattiva di esche alimentari; inoltre, si registra anche l'uso di antagonisti naturali (artropodi e nematodi). Si riportano alcuni esempi di applicazioni in Sicilia di tecniche ecosostenibili di difesa su vite e su colture ortive protette.
\end{abstract}

Parole chiave: prodotti fitosanitari ecosostenibili, feromoni, antagonisti naturali

\section{Biological agents and biotechnological tools for pest control}

\section{Summary}

Over the last few decades, the pest control strategies have progressively focused towards integrated pest management, limiting the use of the chemical control and enhancing the use of biological agents. Scientific evidences in human nutrition and social awareness ask for fruits and vegetables productions with low chemical residues. In this context, research promoted development and marketing of effective plant protection products with a more favorable eco-toxicological profile. As regards to the insecticides, some insecticide formulations based on plant extracts and microbiological formulations are widely used. Moreover, the sexual pheromones are largely used both in the open field and in protected crops (mating disruption), as well as the "attract \& kill" techniques that exploit the attracting action of food baits; at the same time, the use of natural enemies (arthropods and nematodes) is also widely applied. Applications of eco-sustainable control strategies on grapes and protected crops in Sicily are here reported.

Key words: eco-sustainable pesticides, pheromones and natural enemies

\footnotetext{
${ }^{\dagger}$ Nota presentata nell'Adunanza pubblica del 15 novembre 2018

*e-mail: dreina@cbceurope.it
} 


\section{Introduzione}

Negli ultimi decenni la difesa dai parassiti animali delle piante si è progressivamente accostata verso tecniche di difesa integrata, limitando pertanto l'uso prevalente del mezzo chimico a vantaggio di tecniche di lotta alternative preferendo, laddove possibile, l'impiego di agenti e mezzi biologici. Alla luce delle recenti acquisizioni scientifiche in campo nutrizionistico e soprattutto a seguito di una maggiore consapevolezza sociale, si registrano oramai specifiche richieste da parte della GDO (Grande Distribuzione Organizzata) legate soprattutto all'esigenza di limitare la presenza di residui chimici nelle produzioni ortofrutticole commercializzate. Ciò ha pertanto promosso la ricerca, lo sviluppo e la commercializzazione di prodotti fitosanitari parimenti efficaci ma con un profilo eco-tossicologico più favorevole e preferibilmente ammessi in agricoltura biologica.

\section{Principali agenti biologici e mezzi biotecnici}

Ausiliari - L'uso in agricoltura di antagonisti naturali (artropodi parassitoidi e predatori, nematodi entomopatogeni) per il controllo degli insetti dannosi, rappresenta una delle tecniche primariamente più studiate e che hanno incoraggiato studiosi di numerosi paesi a cimentarsi nella valorizzazione dei sistemi di antagonismo naturale applicato agli agroecosistemi. Attualmente le applicazioni più rilevanti si registrano in orticoltura (controllo di afidi, tripidi e aleurodidi su solanacee, cucurbitacee e fragola), ma anche in frutticoltura (Antocoridi contro la Psilla del pero), nonché su agrumi e vite (Imenotteri parassitoidi e Coleotteri predatori contro varie cocciniglie). L'impiego di ausiliari non necessita di alcuna registrazione ministeriale. Risulterà invece sempre più determinante in futuro la presenza di Biofabbriche di produzione nelle vicinanze delle aree di effettivo impiego, allo scopo di contenere al massimo i tempi di consegna e garantire pertanto materiale il più attivo possibile.

Agenti microbiologici - Anche vari agenti microbiologici (virus, batteri, funghi entomopatogeni, lieviti) ad attività insetticida vengono regolarmente adoperati oggi sia in pieno campo che in ambiente protetto (serre) quali mezzi di lotta contro insetti ed altri organismi (batteri, funghi) dannosi alle piante coltivate. Essi sono preliminarmente registrati al pari di altri prodotti fitosanitari presso il Ministero della Salute prima di essere messi in commercio. La lotta microbiologica, ovvero l'uso di microrganismi contro le avversità delle colture, si può considerare nata in Italia, da quando Agostino Bassi nel 1835 segnalò per la prima volta in modo scientifico una malattia sull'insetto Bombyx mori L. chiamata "Mal del Segno" e causata dal fungo Beauveria bassiana (Bals.-Criv.) Vuill.

Allo stato attuale sono registrati in Italia i seguenti agenti microbiologici ad attività insetticida: Adoxophyes orana GV (Virus attivo contro le larve di Adoxophyes orana), Bacillus thuringiensis sottospecie kurstaki e aizawai (Batterio attivo contro larve di Lepidotteri), Beauveria bassiana (Fungo entomopatogeno attivo contro acari, tripidi, aleurodidi, elateridi, tefritidi), Cydia pomonella GV (Virus attivo contro le larve di Cydia pomonella), Lecanicillium muscarium (Fungo attivo contro tripidi e aleurodidi), Metarhizium anisopliae (Fungo attivo contro alcuni insetti terricoli), Helicoverpa armigera NPV (Virus attivo contro le larve di Helicoverpa armigera), Paecilomyces fumosoroseus (Fungo attivo contro forme giovanili di aleirodidi), Spodoptera littoralis NPV (Virus attivo contro larve di Spodoptera littoralis).

Estratti vegetali - Per il controllo biologico degli insetti dannosi, trovano ampio impiego anche alcuni formulati insetticidi a base di estratti vegetali (piretrine, azadiractina), regolarmente registrati come prodotti fitosanitari presso il Ministero della Salute.

Le piretrine sono estratti dai capolini di varie composite, soprattutto da Chrysanthemum cinerariifolium (Trevir.) Vis.. Essi rappresentano l'insetticida biologico più utilizzato con un consumo mondiale di oltre $200.000 \mathrm{~kg}$. Agiscono rapidamente per contatto con un "effetto abbattente", ma 
sono sensibili agli agenti di degradazione naturale, quali soprattutto la luce. Risultano attive nei confronti di molti insetti, afidi in modo particolare [1].

L'azadiractina è un estratto vegetale ottenuto dall'albero del Neem (Azadirachta indica A. Juss.), una pianta della famiglia delle Meliacee originaria dell'India che produce piccoli frutti giallastri con uno o più semi. In India la pianta viene utilizzata da migliaia di anni per diversi scopi (cosmetico, medicinale, controllo insetti dannosi, dentifricio, tonico, ecc.). Nel $1959 \mathrm{H}$. Schmutterer, durante un viaggio in Sudan per trovare soluzioni alle pullulazioni di cavallette, notò che l'unica pianta non attaccata era quella del Neem; questo stimolò il suo interesse a cercarne le motivazioni scientifiche. I semi (assieme alle foglie) sono la parte più interessante per l'estrazione dei principi attivi ad azione insetticida. L'estratto di olio di neem è composto da un cocktail di limonoidi, sebbene l'azione insetticida è dovuta principalmente all' azadiractina. L'attività insetticida è complessa, manifestando un meccanismo chitino-inibitore (azione IGR-simile), nonché di repellenza e fagorepellenza; interferisce inoltre negativamente con la fitness biologica dell'organismo bersaglio riducendone la fecondità e l'ovideposizione. L'azadiractina è attiva su afidi, Psilla del melo, aleirodi, Dorifora della patata, minatori fogliari, nottue, tripidi [2].

Mezzi biotecnici - Ad analogo procedimento normativo di registrazione ministeriale sono soggette le applicazioni di feromoni sintetici usati su larga scala sia in pieno campo che, più recentemente, anche in coltura protetta (confusione e disorientamento sessuale), così come le tecniche "attract \& kill" che sfruttano l'azione attrattiva di esche alimentari.

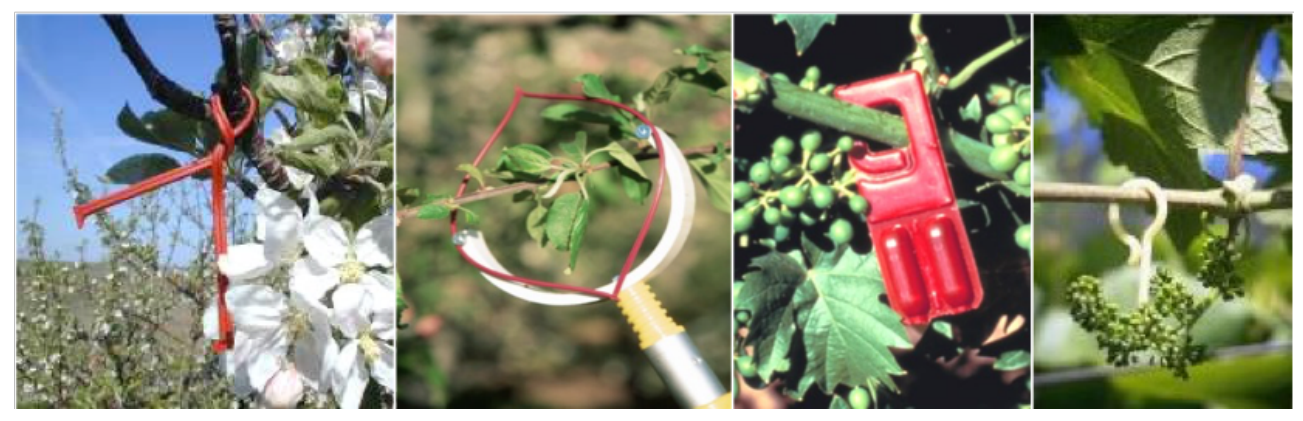

Figura 1: Esempi di erogatori per la confusione e il disorientamento sessuale.

La "confusione sessuale" è un metodo di lotta a carattere preventivo. Attraverso l'immissione nell'ambiente con appositi diffusori dell'analogo di sintesi del feromone sessuale prodotto dalle femmine della specie bersaglio. Questa tecnica mira a creare una massiccia concentrazione di feromone nell'ambiente trattato in grado di mascherare la scia feromonica emessa dalle femmine per attrarre i maschi prima dell'accoppiamento. Viene così inibita la capacità dei maschi di rintracciare le femmine, con conseguente riduzione del numero degli accoppiamenti e della popolazione dell'insetto bersaglio [3, 4]. I diffusori impiegati per la confusione sessuale sfruttano quindi principi di natura chimico-fisica e grande importanza risiede pertanto nella loro tecnologia costruttiva (Figg. 1-2). 

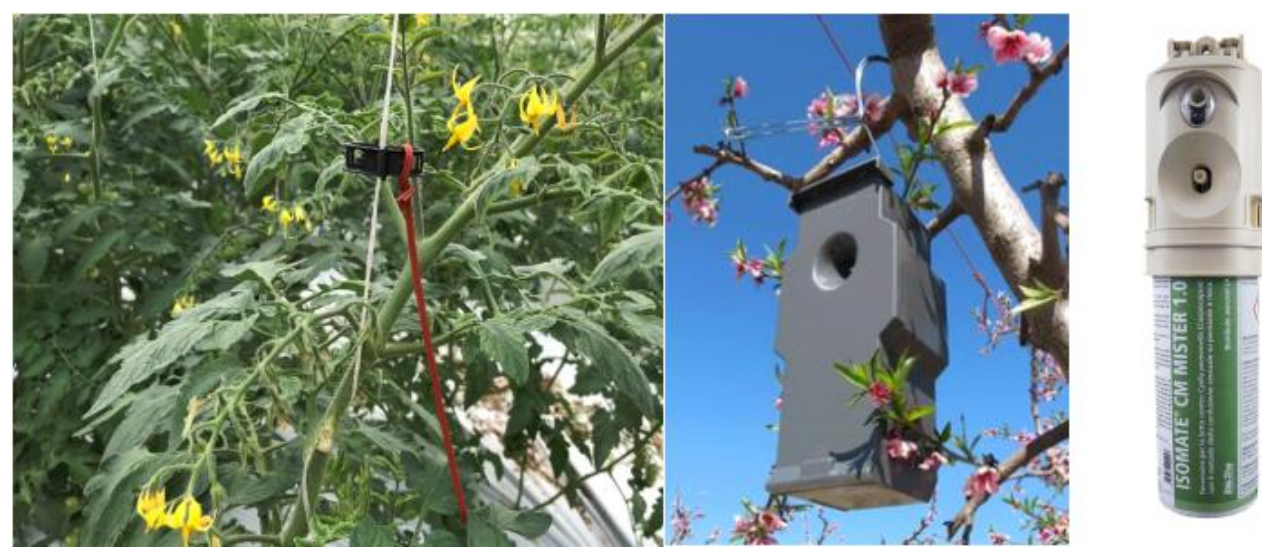

Figura 2: Erogatore per la confusione sessuale contro Tuta absoluta (Meyrick) su pomodoro da mensa in coltura protetta (a sinistra); erogatori aerosol per la confusione sessuale contro Cydia molesta (al centro) e Carpocapsa (a destra).

Allo stato attuale tali sistemi di controllo trovano ampia diffusione in Italia nell'ambito dei principali Lepidotteri dannosi alle Drupacee (Anarsia, Cydia), Pomacee (Carpocapsa, Ricamatori, Rodilegno giallo), Vite (Tignola e Tignoletta) e Pomodoro (Tignola).

Da segnalare il recente sviluppo su larga scala della tecnica "Aerosol", capace di ripetere il segnale delle femmine della specie target attraverso pochi erogatori per ettaro (2-4/ha) (Fig. 2). In Italia trovano già applicazione gli aerosol contro la Carpocapsa delle pomacee e la Tignoletta dell'uva. Da segnalare infine il recente sviluppo applicativo del metodo della confusione sessuale anche su cocciniglie, ed in particolare sulla cocciniglia cotonosa della vite, mentre sono tuttora in fase sperimentale le applicazioni su alcune cocciniglie degli agrumi.

\section{Applicazioni}

Solo a scopo esemplificativo, si riporta un recente successo applicativo in Sicilia caratterizzato dall'impiego sinergico di più tecniche biologiche di difesa che si sono dimostrate di gran lunga più efficaci rispetto ad obsoleti approcci basati unicamente sull'impiego di mezzi chimici.

$\mathrm{Su}$ vite è stato validato il controllo delle infestazioni dei principali fitofagi della coltura, quali la Tignoletta dell'uva [(Lobesia botrana (Denis e Schiffermüller)] (Lepidoptera: Tortricidae) e la Cocciniglia cotonosa (Planococcus ficus (Signoret)] (Rhynchota: Pseudococcidae), mediante l'integrazione di ausiliari, tecniche di confusione sessuale e prodotti fitosanitari biologici. Più in dettaglio, il controllo della Tignoletta si basa innanzitutto sull'applicazione dei sistemi di confusione sessuale: determinante risulta la distribuzione uniforme degli erogatori, nonché l'applicazione tempestiva ad inizio primavera in modo che l'erogazione del feromone sessuale femminile sia già attiva in campo in coincidenza del primo volo dei maschi della specie (in genere a fine marzo). I successivi rilevamenti in fioritura sulla prima generazione della specie saranno determinanti allo scopo di valutare la popolazione presente in campo (percentuale di nidi sui grappoli in fioritura). In funzione dell'eventuale superamento della soglia considerata di danno, si valuterà pertanto l'integrazione successiva di uno o più interventi insetticidi in seconda ed eventualmente anche in terza generazione. A tale scopo, risulta strategico l'impiego di formulati insetticidi a base di " $\mathrm{Ba}$ cillus thuringiensis", attivo contro le larve neonate della Tignoletta [e con profilo tossicologico assolutamente favorevole] [5]. Il controllo della Cocciniglia si basa oramai anch'esso sull'applicazione tempestiva della confusione sessuale. Considerando tuttavia il fatto che la femmina di $P$. ficus sverna già fecondata, può risultare utile nei primi anni di impiego della confusione sessuale l'integrazione sulle forme giovanili della prima generazione di applicazioni insetticide anch'esse biologiche (Azadiractina, appena registrata su tale target), ovvero anche del rilascio in campo degli ausiliari Anagyrus pseudococci (Grlt.) e Cryptolaemus montrouzieri (Muls.), rispettivamente "parassitoide" attivo a bassa densità dell'ospite e "predatore" attivo invece sui focolai della cocciniglia. 
L'esempio riportato rappresenta uno dei più recenti successi del controllo biologico in Italia sulla vite - coltura strategica sull'intero territorio nazionale sia in ambito viti-vinicolo che sui moderni impianti di uva da tavola del sud Italia. Sulla stessa coltura ulteriori futuri sviluppi sono rappresentati dalle attive ricerche su applicazioni a carattere "elettrico" che hanno l'obiettivo di emettere segnali vibrazionali che riproducono e diffondono segnalazioni specifiche emesse dalle dannose Cicaline Scaphoideus titanus (Ball.) ed Empoasca vitis (Goet.), interferendo nella comunicazione tra i sessi ed impedendone o ritardandone notevolmente l'accoppiamento.

\section{Considerazioni conclusive}

L'Italia ha storicamente avuto un ruolo strategico di capofila nella ricerca scientifica sul controllo biologico degli insetti dannosi in agricoltura, sebbene solo recentemente il mondo applicativo si sia seriamente accostato a tali tecniche, pressato dalle precise richieste da parte della GDO in termini di bassa o nulla residualità di presidi chimici nell'ortofrutta commercializzata. In gran parte dei paesi europei, così come nelle aree agricole più avanzate del Nord Africa, la diffusione di tali tecniche risulta paradossalmente di gran lunga superiore a quella registrata in Italia, grazie ad una generale maggiore sensibilità del mondo civile - soprattutto quello nord-europeo - che è riuscito ad influenzare le scelte tecniche della realtà produttiva a favore di metodologie biologiche e più ecosostenibili.

Il virtuoso percorso intrapreso in Italia sembra fortunatamente ben avviato, così come il processo di progressiva eliminazione a livello comunitario dei prodotti fitosanitari più tossici, sia per l'uomo che per l'ambiente, prima ampiamente utilizzati.

Tale percorso tuttavia non è tuttora privo di evidenti limiti, anche strutturali. Dal punto di vista legislativo si registrano soprattutto nel nostro paese non poche carenze che determinano il facile ingresso nel mercato di mezzi tecnici apparentemente biologici, in realtà privi addirittura dei più elementari principi normativi della fitoiatria. Inoltre, il mondo professionale appare ancora non completamente formato e pronto per un cambiamento tecnico così significativo. Tuttavia, la pressante leva commerciale impressa dalla GDO unita alla forte sensibilizzazione di una fascia sempre più ampia e attiva dell'opinione pubblica sembrano fornire la giusta pressione per un reale e definitivo cambiamento.

\section{Riferimenti bibliografici}

Fabbri, L. 2016, Le piretrine naturali, una risorsa strategica. Colture Protette 11, 18-20.

Marzocchi, L. 2016, L'Azadiractina, una risorsa per proteggere le colture. Colture Protette 3, 8-11.

Cavagnero, M., Pavarino, A. 2012, Cosa influenza il rilascio di feromoni contro le tignole della vite. L'Informatore Agrario 28, 67-70.

Anfora, G., Ioratti, C. 2016, La confusione sessuale per la lotta alle tignole. Vigne, vini qualità $3,16-18$.

Bagnoli, B., Lucchi, A., Pollini A. 2009, Contro le tignole della vite si interviene sulla $2 a$ generazione. L'Informatore Agrario 22, 9-11. 